\title{
Sliding Mode Observer-Based Finite Time Control Scheme for Frequency Regulation and Economic Dispatch in Power Grids
}

\author{
Gianmario Rinaldi ${ }^{1}$, Prathyush P. Menon ${ }^{1}$, Christopher Edwards ${ }^{2}$ and Antonella Ferrara ${ }^{3}$
}

\begin{abstract}
In this paper a novel Sliding Mode (SM) observer-based scheme is proposed to achieve frequency regulation and Economic Dispatch (ED) in power grids composed of an interconnection of generators and load buses. The ED problem is addressed in two steps. Assuming only the voltage phase angles are measured, in the first step a network of heterogeneous SM observers, suitably interconnected in a distributed fashion, is created to estimate both frequency deviations and unknown power levels associated with each bus. In the second step, the observer scheme is coupled with a SM control strategy which is able to reach the optimal value of the control input in each generator bus in finite time. The scheme is assessed via the IEEE 39 bus benchmark, and a comparison with existing control methods is provided.
\end{abstract}

Index Terms

Observers for nonlinear systems; Variable-structure/sliding-mode control; Power systems.

\section{INTRODUCTION}

$\mathbf{T}$ He frequency in power grids must be kept close to its nominal value in order to prevent damage to facilities, widespread outages, blackouts and degradation in power quality [1]. A hierarchical control architecture has been traditionally employed for the purpose of frequency control. This scheme is composed of three levels - from fast to slow in time-scale: a primary droop controller, a Proportional Integral (PI) secondary controller, and a tertiary controller devoted to Economic Dispatch (ED) [2], [3]. The growing integration of distributed renewable energy-based power sources gives rise to larger power mismatches between generation and consumption. Such a scenario calls for the design of faster, more efficient, and more reliable control schemes. A detailed review of recent advances in frequency control schemes can be found, for example, in the literature survey [4]. While guaranteeing a balance between power generation and consumption, it is often necessary to minimise the aggregated generation cost, thus fulfilling the economic constraints. This problem is known as ED [3], [5]. Recently, it has been argued that there is a need for breaking the conventional control hierarchy described above and for directly introducing ED in the secondary frequency control architecture [6]. Different approaches have been formulated for ED in power grids. For example, in [7] both an incremental passivity technique and an internal-model-based controller architecture have been employed to design distributed controllers for frequency regulation and ED in power networks. In [8] a linearised model of a power network has been used as a starting point to design a distributed primary control scheme, which also accounts for the cost associated with frequency-dependent load. The approach was extended in [6], with the use of a distributed PI optimal controller stabilising power networks modelled by a Differential Algebraic Equations (DAEs) structure, and in [9], in which a plug-and-play architecture was introduced. Recently, in [10] an optimisation architecture has been formulated for ED in smart power grids, where the optimisation problem was solved by only using local information available at the generator level.

Sliding Mode (SM) control techniques represent promising strategies for the stabilisation of nonlinear (uncertain) systems, due to their capability to both reach in finite time a selected sliding surface, and to reject bounded disturbances appearing in the input channels of the system [11]. SM control architectures have already been successfully applied to control power systems. For example, in [12], a passivity-based approach was formulated for frequency regulation and ED in a power network, which included a linearised model of the turbine-governor dynamics. The power flow throughout the transmission line follows the nonlinear power flow formulation. In [13], a more detailed hydro-power plant model has been employed, and a linearised DC load flow method was used for power flow. In order to reduce the number of required sensors for the implementation of the control scheme, SM state observers can be adopted to dynamically and robustly estimate the unmeasured state variables. These dynamical state estimators are also able to reconstruct unknown inputs, faults and disturbances, to enhance the monitoring and

\footnotetext{
1 Gianmario Rinaldi and Prathyush P. Menon are with the Centre for Future Clean Mobility, University of Exeter, Exeter, United Kingdom. G.Rinaldi3@exeter.ac.uk, P.M.Prathyush@exeter.ac.uk

${ }^{2}$ Christopher Edwards is with College of Engineering, Mathematics, and Physical Sciences, University of Exeter, Exeter, United Kingdom.

C.Edwardseexeter.ac.uk.

3 Antonella Ferrara is with Department of Electrical, Computer and Biomedical Engineering, University of Pavia, Pavia, Italy. antonella.ferrara@unipv.it.

$\star$ This is the final version of the accepted paper included in the IEEE Transactions on Control Systems Technology, 2021.
} 
Table I

List OF Symbols AND VARIABLES USED IN THE PAPER.

\begin{tabular}{r|l}
\hline Symbol & Meaning \\
\hline$\theta_{i}$ (rad) & voltage phase angle \\
$\omega_{i}$ (p.u.) & frequency deviation \\
$p_{i}$ (p.u.) & unknown power injection or demand \\
$u_{i}$ (p.u.) & control variable \\
\hline$D_{i}$ (p.u.) & droop control coefficient \\
$M_{i}$ (p.u.) & generator inertia \\
$\omega^{\star}$ (p.u.) & nominal grid frequency \\
\hline
\end{tabular}

thus the control of the system [14], [15]. In [16] the control scheme proposed in [12] has been coupled with a network of higher order SM observers to estimate the frequency deviation and the governor output power variation. Relatively few works have dealt with estimator schemes for structure-preserving power grid models (see, e.g., [17], [18], [19]). The structure-preserving approach aims to explicitly model each component of the network and it is shown to be more accurate, thus considering the expression of electrical power flow in each edge (i.e., in each power transmission line) [20].

Main Contribution: In this paper a novel observer-based SM control scheme is proposed to achieve both frequency regulation and ED for structure-preserving power grids. A power grid composed of an interconnection of generator (controllable) buses and frequency-dependent load buses is considered. Assuming that only the voltage phase angles are measured via Phasor Measurement Units (PMUs) [2], a distributed heterogeneous SM observer scheme is formulated to estimate in finite time the frequency deviation in the generator buses and the unknown power associated with all the buses. Then, a reference maker node is introduced, which has the task to collect the estimates coming from the observers and generate the optimal value for the marginal cost. A SM control scheme is introduced to drive the control input to its optimal value in finite time. In contrast to [7], [16], and [12], in which the power demand is treated as an unknown input, in the present approach a strategy is presented to estimate the power demand in finite time, thus reducing the complexity of the control scheme. Furthermore, the present scheme is able to perform unknown input state estimation in finite time, and not asymptotically, as typically happens if UI Luenberger observers are employed [21]. In contrast to [16], [22], and [23], in which the optimal value of the marginal generation cost is asymptotically achieved, the proposed scheme is able to reach the optimal marginal cost in finite time.

Notation: The following standard notation is employed throughout the paper. For a given continuous-time signal $x, \hat{x}$ represents its estimate. For a given vector or matrix $X$, the expression $X^{T}$ defines its transpose. The symbol 1 denotes a column vector of appropriate dimensions with all its entries set equal to 1 . The function $\operatorname{sign}(\cdot)$ represents the sign function. For a given matrix $X$, the expression $X_{(y \times z)}$ specifies that $X \in \mathbb{R}^{y \times z}$, where $y$ and $z$ are positive integers. The expressions $\operatorname{Col}(\cdot)$ and $\operatorname{Diag}(\cdot)$ define a column vector and a diagonal matrix, respectively. The expression $X \succ 0$ denotes that the matrix $X$ is positive definite. The gradient of a given function $f(\cdot)$ is defined as $\nabla f(\cdot)$. The symbol $\|\cdot\|$ represents the Euclidean norm. For a given set $\mathcal{X}$, the expression $|\mathcal{X}|$ denotes its cardinality.

\section{POWER GRID DESCRIPTION}

A structure-preserving power grid model can be described by a connected and undirected graph $G(\mathcal{B}, \mathcal{E})$, with the set of buses (commonly called also nodes) $\mathcal{B}=\{1, \ldots,|\mathcal{B}|\}$, and the set of edges $\mathcal{E} \subseteq \mathcal{B} \times \mathcal{B}$ [20]. The set of buses $\mathcal{B}$ will be partitioned into the so-called generation buses $\mathcal{G}=\{1, \ldots,|\mathcal{G}|\}$ and frequency-dependent load buses $\mathcal{L}=\{|\mathcal{G}+1|, \ldots,|\mathcal{B}|\}$, such that $\mathcal{B}=\mathcal{G} \cup \mathcal{L}$. The reader is referred to Table I for the physical interpretation of the state variables and model parameters. The symbol $\mathcal{N}_{i}$ denotes the set of buses directly connected to the $i$-th bus via edges. By arbitrarily labelling with ${ }^{\prime}+^{\prime}$ and ${ }^{\prime}-^{\prime}$ the positive and the negative ends of an edge, the incident matrix $B \in \mathbb{R}^{|\mathcal{B}| \times|\mathcal{E}|}$, which encapsulates the topology of the grid, is defined as:

$$
B_{i j}= \begin{cases}1 & \text { if } i \text { is the positive end of } j \\ -1 & \text { if } i \text { is the negative end of } j \\ 0 & \text { otherwise }\end{cases}
$$

The grid dynamics are described by the following differential equations, which represents a structure-preserving model of generators and loads interconnected by high-voltage power transmission lines [24] ${ }^{1}$

$$
\begin{aligned}
\dot{\theta}_{i} & =\omega_{i} & & i \in \mathcal{B} \\
M_{i} \dot{\omega}_{i} & =-D_{i} \omega_{i}+p_{i}+u_{i}-\sum_{j \in \mathcal{N}_{i}} P_{i j}\left(\theta_{i}, \theta_{j}\right) & & i \in \mathcal{G} \\
0 & =-D_{i} \omega_{i}+p_{i}-\sum_{j \in \mathcal{N}_{i}} P_{i j}\left(\theta_{i}, \theta_{j}\right) & & i \in \mathcal{L}, \\
y_{i} & =\theta_{i}, & & i \in \mathcal{B} .
\end{aligned}
$$

\footnotetext{
${ }^{1}$ The time dependency is omitted thought the manuscript for the sake of readability.
} 
Remark 1 The signal $P_{i j}(\cdot)$ in (2) represents the total electric power flowing from the $i$-th to the $j$-th bus. If the power transmission lines are considered lossless and the voltage phase angles at the terminals of the line are sufficiently close to each other, then [3], [2]

$$
P_{i j}\left(\theta_{i}, \theta_{j}\right):=\gamma_{i j} \sin \left(\theta_{i}-\theta_{j}\right)
$$

The positive parameter $\gamma_{i j}$ depends on the power transmission line susceptance and on the voltage magnitude at each terminal of the line [3]. For the stability analysis of the proposed observer-based control strategy, it is assumed that $\gamma_{i j}$ is known and slow time-varying. If $\gamma_{i j}$ is uncertain or unknown, it follows that an analytical expression for $P_{i j}(\cdot)$ cannot be used. However, one can still measure $P_{i j}(\cdot)$ via a PMU installed at each terminal of the power transmission line [2] and the approach proposed in the paper is still applicable.

Assumption 1 The unknown power injection or demand vector $p:=\operatorname{Col}\left(p_{1}, \ldots, p_{|\mathcal{B}|}\right) \in \mathbb{R}^{|\mathcal{B}|}$ is bounded, with a bounded first time derivative. Furthermore, it is assumed the bounds are known and the scalars $\Lambda_{p}$ and $\Lambda_{\dot{p}}$ as such that:

$$
\|p\| \leq \Lambda_{p} \quad\|\dot{p}\| \leq \Lambda_{\dot{p}}
$$

\section{Distributed Observers Design and Unknown Power Demand Reconstruction}

In this section, a network of SM observers, suitably interconnected in a distributed fashion, is employed for both dynamically estimating the frequency deviation in each generation bus and reconstructing the unknown power injection and demand in finite time.

\section{A. Third Order SM Observers for Generators}

Proposition 1 Given the generator dynamics (2), and Assumption 1, the third order SM observer

$$
\left.\begin{array}{rl}
\dot{\hat{\theta}}_{i}= & k_{g_{i}}\left|e_{\theta_{i}}\right|^{2 / 3} \operatorname{sign}\left(e_{\theta_{i}}\right)+a_{i} e_{\theta_{i}}+\hat{\omega}_{i} \\
\dot{\hat{\omega}}_{i}= & k_{2_{g_{i}}}\left|e_{\theta_{i}}\right|^{1 / 3} \operatorname{sign}\left(e_{\theta_{i}}\right)+a_{i} \hat{\omega}_{i}+a_{i}^{2} e_{\theta_{i}} \\
& +\frac{1}{M_{i}}\left(u_{i}-\sum_{j \in \mathcal{N}_{i}} P_{i j}\left(y_{i}, y_{j}\right)\right) \\
& +k_{g_{g_{i}}} a_{i}\left|e_{\theta_{i}}\right|^{2 / 3} \operatorname{sign}\left(e_{\theta_{i}}\right)+\hat{z}_{i} \\
\dot{\hat{z}}_{i}= & k_{3_{g_{i}}} \operatorname{sign}\left(e_{\theta_{i}}\right)
\end{array}\right\} \forall i \in \mathcal{G}
$$

where: $\hat{\theta}_{i}$ is an estimate of $\theta_{i}, \hat{\omega}_{i}$ is an estimate of $\omega_{i}, \hat{z}_{i}$ is an auxiliary observer variable, $a_{i}:=-D_{i} / M_{i}, e_{\theta_{i}}:=\theta_{i}-\hat{\theta}_{i}, \hat{z}_{i}$ is an auxiliary variable, and $k_{g_{i}}, k_{2_{g_{i}}}, k_{3_{g_{i}}}$ are design constants, estimates the frequency deviation and the unknown power demand

$$
\hat{p}_{i}=M_{i} \hat{z}_{i}, \forall i \in \mathcal{G}
$$

in finite time.

Proof: Subtracting (5) from the generator dynamics in (2) yields the error dynamics for each generator as

$$
\left.\begin{array}{rl}
\dot{e}_{\theta_{i}}= & -k_{1_{g_{i}}}\left|e_{\theta_{i}}\right|^{2 / 3} \operatorname{sign}\left(e_{\theta_{i}}\right)-a_{i} e_{\theta_{i}}+e_{\omega_{i}} \\
\dot{e}_{\omega_{i}}= & -k_{2_{g_{i}}}\left|e_{\theta_{i}}\right|^{1 / 3} \operatorname{sign}\left(e_{\theta_{i}}\right)+a_{i} e_{\omega_{i}} \\
& -a_{i}^{2} e_{\theta_{i}}-k_{g_{g_{i}}} a_{i}\left|e_{\theta_{i}}\right|^{2 / 3} \operatorname{sign}\left(e_{\theta_{i}}\right) \\
& -\hat{z}_{i}+\frac{p_{i}}{M_{i}} \\
\dot{z}_{i}= & k_{g_{g_{i}}} \operatorname{sign}\left(e_{\theta_{i}}\right)
\end{array}\right\} \forall i \in \mathcal{G}
$$

where $e_{\omega_{i}}:=\omega_{i}-\hat{\omega}_{i}$. It is now possible to define $\bar{e}_{\omega_{i}}:=e_{\omega_{i}}-a_{i} e_{\theta_{i}}$, and rewrite (7) as follows:

$$
\left.\begin{array}{rl}
\dot{e}_{\theta_{i}}= & -k_{1_{g_{i}}}\left|e_{\theta_{i}}\right|^{2 / 3} \operatorname{sign}\left(e_{\theta_{i}}\right)-a_{i} e_{\theta_{i}}+e_{\omega_{i}} \\
\dot{\bar{e}}_{\omega_{i}}= & -k_{g_{g_{i}}}\left|e_{\theta_{i}}\right|^{1 / 3} \operatorname{sign}\left(e_{\theta_{i}}\right)+a_{i} e_{\omega_{i}} \\
& -a_{i}^{2} e_{\theta_{i}}-k_{g_{g_{i}}} a_{i}\left|e_{\theta_{i}}\right|^{2 / 3} \operatorname{sign}\left(e_{\theta_{i}}\right) \\
& -\hat{z}_{i}+\frac{p_{i}}{M_{i}}+k_{g_{g_{i}}} a_{i}\left|e_{\theta_{i}}\right|^{2 / 3} \operatorname{sign}\left(e_{\theta_{i}}\right) \\
& +a_{i}^{2} e_{\theta_{i}}-a_{i} e_{\omega_{i}} \\
\dot{\hat{z}}_{i}= & k_{3_{g_{i}}} \operatorname{sign}\left(e_{\theta_{i}}\right)
\end{array}\right\}
$$

After straightforward algebraic simplifications in (8), and by introducing another auxiliary error variable as

$$
e_{z_{i}}:=-\hat{z}_{i}+\frac{p_{i}}{M_{i}}, \forall i \in \mathcal{G}
$$


the following error dynamics are obtained:

$$
\left.\begin{array}{rl}
\dot{e}_{\theta_{i}} & =-k_{1_{g_{i}}}\left|e_{\theta_{i}}\right|^{2 / 3} \operatorname{sign}\left(e_{\theta_{i}}\right)+\bar{e}_{\omega_{i}} \\
\dot{\bar{e}}_{\omega_{i}} & =-k_{2_{g_{i}}}\left|e_{\theta_{i}}\right|^{1 / 3} \operatorname{sign}\left(e_{\theta_{i}}\right)+e_{z_{i}} \\
\dot{e}_{z_{i}} & =-k_{3_{g_{i}}} \operatorname{sign}\left(e_{\theta_{i}}\right)+\frac{\dot{p}_{i}}{M_{i}}
\end{array}\right\} \forall i \in \mathcal{G}
$$

The system in equations (10) is in the canonical form of the third order SM observer error dynamics in [25]. From Assumption 1, it follows that $\left|\dot{p}_{i} / M_{i}\right| \leq \tilde{\Delta}_{i}$, where $\tilde{\Delta}_{i}$ is a known positive constant. Following [26], a Lyapunov function candidate in the form of

$$
\begin{aligned}
V_{g_{i}}\left(e_{\theta_{i}}, \bar{e}_{\omega_{i}}, e_{z_{i}}\right)= & \beta_{1 i}\left|e_{\theta_{i}}\right|^{5 / 3}-\beta_{2 i} e_{\theta_{i}} \bar{e}_{\omega_{i}}+\beta_{3 i}\left|\bar{e}_{\omega_{i}}\right|^{5 / 2} \\
& -\beta_{4 i} \bar{e}_{\omega_{i}}\left|e_{z_{i}}\right|^{3} \operatorname{sign}\left(e_{z_{i}}\right)+\beta_{5 i}\left|e_{z_{i}}\right|^{5}
\end{aligned}
$$

can be employed to show that in the error dynamics (10) the origin is finite time stable, where $\beta_{1 i}, \ldots, \beta_{5 i}$ are suitably chosen positive constants. In order to tune the design constants, let

$$
\tilde{L}_{i}=\tilde{\Delta}_{i} / \tilde{\Delta}_{i 0}
$$

where $\tilde{\Delta}_{i 0}$ is a known constant. In particular, following the methodology in [26], $\tilde{\Delta}_{i 0}=0.0081$. Then, if $\left[\bar{k}_{1_{g_{i}}}, \bar{k}_{2_{g_{i}}}, \bar{k}_{3_{g_{i}}}\right]^{T}$ are the triplet of the design constants tuned in [26] for the unperturbed case (i.e., for the case when $\dot{p}_{i} / M_{i}=0$ ), it can be shown [26] that $\left[k_{g_{i}}, k_{2_{g_{i}}}, k_{3_{g_{i}}}\right]^{T}$ can be chosen according to:

$$
\left[k_{1_{i}}, k_{2_{g_{i}}}, k_{3_{g_{i}}}\right]^{T}=\operatorname{Diag}\left(\tilde{L}_{i}^{1 / 3}, \tilde{L}_{i}^{2 / 3}, \tilde{L}_{i}\right)\left[\left[\bar{k}_{1_{g_{i}}}, \bar{k}_{2_{g_{i}}}, \bar{k}_{3_{g_{i}}}\right]^{T}\right.
$$

The numerical representation of the tuning rules (13) introduced in [27] can be shown to be:

$$
\begin{aligned}
& k_{3_{g_{i}}}>\tilde{\Delta}_{i} \\
& k_{2_{g_{i}}}=5.3 k_{3_{g_{i}}}^{2 / 3} \\
& k_{1_{g_{i}}}=3.34 k_{3_{g_{i}}}^{1 / 3} .
\end{aligned}
$$

Note that the gain $k_{3_{i}}$ is the first to be tuned to compensate the effect of $\dot{p}_{i} / M_{i}$. Furthermore, if the vector of the initial conditions of (10) is $e_{0_{g_{i}}}:=\operatorname{Col}\left(e_{\theta_{i}}(0), \bar{e}_{\omega_{i}}(0), e_{z_{i}}(0)\right)$, then the dynamics converge to the origin in a finite time $T_{e_{g_{i}}}$, which is upper-bounded according to:

$$
T_{e_{g_{i}}} \leq \frac{V_{g_{i}}^{1 / 5}\left(e_{g_{i}}\right)}{c_{g_{i}}}
$$

where $c_{g_{i}}$ is a positive constant. Therefore, a sliding motion is characterized by the following conditions

$$
e_{\theta_{i}}=\bar{e}_{\omega_{i}}=e_{\omega_{i}}=e_{z_{i}}=0, \forall i \in \mathcal{G}
$$

which are enforced in finite time. Two objectives are achieved once the sliding motion (16) occurs: $i$ ) it is possible to correctly estimate the frequency deviation $\omega_{i}$, since $\left.e_{\omega_{i}}=\omega_{i}-\hat{\omega}_{i}=0, i i\right)$ since $e_{z_{i}}=0$, according to (9), the condition (6) is satisfied in finite time. This proves the proposition.

\section{B. Super-Twisting SM Observers for Loads}

Inspired by [28], the following proposition holds for each load bus $i \in \mathcal{L}$.

Proposition 2 Given the load dynamics in (2), and Assumption 1, the following super-twisting SM observer is able to estimate in finite time the unknown power demand $p_{i}$ :

$$
\left.\begin{array}{rl}
\dot{\hat{\theta}}_{i}= & k_{1_{l_{i}}}\left|e_{\theta_{i}}\right|^{1 / 2} \operatorname{sign}\left(e_{\theta_{i}}\right) \\
& -\frac{1}{D_{i}} \sum_{j \in \mathcal{N}_{i}} P_{i j}\left(y_{i}, y_{j}\right)+\hat{\psi}_{i} \\
\dot{\hat{\psi}}_{i}= & k_{2_{l_{i}}} \operatorname{sign}\left(e_{\theta_{i}}\right)
\end{array}\right\} \forall i \in \mathcal{L}
$$

where $\hat{\psi}_{i}$ is an auxiliary variable, $e_{\theta_{i}}:=\theta_{i}-\hat{\theta}_{i}$, and $k_{1_{l_{i}}}, k_{2_{l_{i}}}$ are design constants. An estimate $\hat{p}_{i}$ of the unknown power $p_{i}$ can be obtained by using the following relation:

$$
\hat{p}_{i}=D_{i} \hat{\psi}_{i}, \forall i \in \mathcal{L}
$$


Proof: The error dynamics for each load bus are computed by subtracting (17) from the load dynamics in (2), and they are given by:

$$
\left.\begin{array}{rl}
\dot{e}_{\theta_{i}} & =-k_{l_{l_{i}}}\left|e_{\theta_{i}}\right|^{1 / 2} \operatorname{sign}\left(e_{\theta_{i}}\right)+\frac{p_{i}}{D_{i}}-\hat{\psi}_{i} \\
\dot{\hat{\psi}}_{i} & =k_{2_{i}} \operatorname{sign}\left(e_{\theta_{i}}\right)
\end{array}\right\} \forall i \in \mathcal{L}
$$

It is possible to introduce an auxiliary error variable

$$
e_{\psi_{i}}:=-\hat{\psi}_{i}+\frac{p_{i}}{D_{i}}, \forall i \in \mathcal{L}
$$

thus obtaining the following error dynamics

$$
\left.\begin{array}{rl}
\dot{e}_{\theta_{i}} & =-k_{l_{l_{i}}}\left|e_{\theta_{i}}\right|^{1 / 2} \operatorname{sign}\left(e_{\theta_{i}}\right)+e_{\psi_{i}} \\
\dot{e}_{\psi_{i}} & =-k_{2_{l_{i}}} \operatorname{sign}\left(e_{\theta_{i}}\right)+\frac{\dot{p}_{i}}{D_{i}} .
\end{array}\right\} \forall i \in \mathcal{L}
$$

The system in equation (21) is in the canonical form of a super-twisting SM architecture. Therefore, by tuning the design constants following, for example, the rules in [29], the system in is driven to the origin in a finite time $T_{e_{0_{l_{i}}}}$. It follows that

$$
e_{\theta_{i}}=e_{\psi_{i}}=0, \forall i \in \mathcal{L}
$$

Therefore, exploiting (20), it follows that condition (18) holds in finite time.

Remark 2 The proposed third order SM observer scheme in (5) and the super-twisting observer in (17) exhibit a distributed structure since they use local voltage phase angles $\left(y_{i}\right)$ and phase angle measurements gathered by the neighbouring buses $\left(y_{j}, j \in \mathcal{N}_{i}\right)$. Furthermore, the power network and the observer network have the same topology. However, if the signal $P_{i j}(\cdot)$ is obtained from the PMU as illustrated in Remark 1, the observers in equations (5) and (17) become fully decentralised.

Remark 3 Fault reconstruction schemes based on SM principles, often require the use of low-pass filters to eliminate the high frequency components and obtain good quality fault estimation [14]. However, such a low-pass filter mechanism is not mandatory for the reconstruction of unknown powers. This is due to the fact that the signal $\hat{z}_{i}$ in (6) and $\hat{\psi}_{i}$ in (18) are continuous, because the order of the observers has been increased by one with respect to the order of the system under observation.

\section{Controllers Design}

\section{A. Preliminaries}

The distributed observer, proposed in (5) and (17), estimates the unknown power demands, both at generators and loads, in finite-time. Making use of this information, the objective of the controller scheme proposed here is to drive the deviation in frequency asymptotically to zero, while the cost of generators operation is minimised.

Assumption 2 Suppose that there exists a reference-maker which collects all the estimates of the unknown powers $p_{i}$ for generators and loads. Therefore, the following column vector can be introduced

$$
\hat{p}:=\operatorname{Col}\left(\hat{p}_{1}, \ldots, \hat{p}_{|\mathcal{B}|}\right),
$$

where $\hat{p} \in \mathbb{R}^{|\mathcal{B}|}$.

The dynamics (2) are rewritten in a compact form as follows:

$$
\begin{aligned}
\dot{\theta} & =\omega \\
M_{\mathcal{G}} \dot{\omega}_{\mathcal{G}} & =-D_{\mathcal{G}} \omega_{\mathcal{G}}+u-\nabla_{\mathcal{G}} U(\theta) \\
0 & =-D_{\mathcal{L}} \omega_{\mathcal{L}}-\nabla_{\mathcal{L}} U(\theta) \\
y & =\theta
\end{aligned}
$$

where $\theta:=\operatorname{Col}\left(\theta_{1}, \ldots, \theta_{|\mathcal{B}|}\right) \in \mathbb{R}^{|\mathcal{B}|}, \omega_{\mathcal{G}}:=\operatorname{Col}\left(\omega_{1}, \ldots, \omega_{|\mathcal{G}|}\right) \in \mathbb{R}^{|\mathcal{G}|}, \omega_{\mathcal{L}}:=\operatorname{Col}\left(\omega_{|\mathcal{G}|+1}, \ldots, \omega_{|\mathcal{B}|}\right) \in \mathbb{R}^{|\mathcal{L}|}, \omega:=\operatorname{Col}\left(\omega_{\mathcal{G}}, \omega_{\mathcal{L}}\right) \in$ $\mathbb{R}^{|\mathcal{B}|}, M_{\mathcal{G}}:=\operatorname{Diag}\left(\left(M_{i}\right)_{i \in \mathcal{G}}\right), M_{\mathcal{G}} \succ 0, D_{\mathcal{G}}:=\operatorname{Diag}\left(\left(D_{i}\right)_{i \in \mathcal{G}}\right), D_{\mathcal{G}} \succ 0, D_{\mathcal{G}} \in \mathbb{R}^{|\mathcal{G}| \times|\mathcal{G}|}, D_{\mathcal{L}}:=\operatorname{Diag}\left(\left(D_{i}\right)_{i \in \mathcal{L}}\right), D_{\mathcal{L}} \succ 0, D_{\mathcal{L}} \in$ $\mathbb{R}^{|\mathcal{L}| \times|\mathcal{L}|} u:=\operatorname{Col}\left(u_{1}, \ldots, u_{|\mathcal{G}|}\right) \in \mathbb{R}^{|\mathcal{G}|}$, and $y:=\operatorname{Col}\left(y_{1}, \ldots, y_{|\mathcal{B}|}\right) \in \mathbb{R}^{|\mathcal{B}|}$. Finally, $U(\theta)$ denotes the so-called open-loop potential energy of the aggregated generator and load buses, which is obtained by making use of (1) and (3), and it is given by [6], [30]:

$$
U(\theta):=\underline{1}^{T} \Gamma\left(1-\cos \left(B^{T} \theta\right)\right)-p^{T} \theta
$$


where $\Gamma=\operatorname{Diag}\left(\gamma_{i j}\right)_{\gamma_{i j} \in \mathcal{E}}, \Gamma \in \mathbb{R}^{|\mathcal{E}| \times|\mathcal{E}|}, p=\operatorname{Col}\left(p_{1}, \ldots, p_{|\mathcal{B}|}\right), p \in \mathbb{R}^{|\mathcal{B}|}$

$$
\nabla U(\boldsymbol{\theta})=\left[\begin{array}{c}
\nabla_{\mathcal{G}} U(\boldsymbol{\theta}) \\
\nabla_{\mathcal{L}} U(\boldsymbol{\theta})
\end{array}\right], \nabla U(\boldsymbol{\theta}) \in \mathbb{R}^{|\mathcal{B}|}
$$

represents the gradient of the potential energy $U(\theta)$ in $(25)$, and the sub-vectors $\nabla_{\mathcal{G}} U(\theta) \in \mathbb{R}^{|\mathcal{G}|}$ and $\nabla_{\mathcal{L}} U(\theta) \in \mathbb{R}^{|\mathcal{L}|}$ represent contributions composed only of the components from the the sets $\mathcal{G}$ and $\mathcal{L}$.

Remark 4 The proposed control acts on the power grid only after the observer error dynamics (10) and (21) convergence to the origin, which takes place in finite time $T_{e_{0}}=\max \left(T_{e_{g_{i}}} \in \mathcal{G}, T_{e_{l_{i}}} \in \mathcal{L}\right)$. Hence, in the analysis which follows:

$$
\begin{aligned}
& \hat{\theta}=\theta \\
& \hat{\omega}=\omega \\
& \hat{p}=p
\end{aligned}
$$

where the estimate vectors $\hat{\theta}:=\operatorname{Col}\left(\hat{\theta}_{1}, \ldots, \hat{\theta}_{|\mathcal{B}|}\right) \in \mathbb{R}^{|\mathcal{B}|}$, and $\hat{\omega}:=\operatorname{Col}\left(\hat{\omega}_{1}, \ldots, \hat{\omega}_{|\mathcal{B}|}\right) \in \mathbb{R}^{|\mathcal{B}|}$. Prior to $T_{e_{0}}$, only the primary frequency control scheme [2] acts on the power grid. This is characterised by the term $-D \omega:=\operatorname{Col}\left(-D_{\mathcal{G}} \omega_{\mathcal{G}},-D_{\mathcal{L}} \omega_{\mathcal{L}}\right) \in \mathbb{R}^{|\mathcal{B}|}$ in (24). This primary control architecture ensures that, for a given constant power demand $\bar{p}$ in each bus, all the frequencies $\omega_{i}$ of the generator buses asymptotically reach the same constant value $\bar{\omega}$, which is a synchronised frequency deviation generally different from zero [3], [2].

The ED optimisation problem is formulated as follows [12], [6], [2]:

$$
\operatorname{ED}\left\{\begin{aligned}
\min _{u, \theta} C(u) & :=\frac{1}{2} u^{T} Q u=\sum_{i \in \mathcal{G}} \frac{1}{2} Q_{i} u_{i}^{2} \\
\text { subject to } & \\
0 & =\underline{1}^{T}\left(u-\nabla_{\mathcal{G}} U(\theta)\right)-\underline{1}^{T} \nabla_{\mathcal{L}} U(\theta) \\
\left|\theta_{i}-\theta_{j}\right| & \leq \alpha_{i j}<\frac{\pi}{2}, \forall i \in \mathcal{B}, j \in \mathcal{N}_{i}
\end{aligned}\right.
$$

where $C(u)$ is a quadratic cost function, $Q=\operatorname{Diag}\left(Q_{1}, \ldots, Q_{|\mathcal{G}|}\right), Q \succ 0, Q \in \mathbb{R}^{|\mathcal{G}| \times|\mathcal{G}|}$ is a known positive definite diagonal matrix; the control vector $u \in \mathbb{R}^{|\mathcal{G}|}=\operatorname{Col}\left(u_{i}\right)$ has to be designed; and $\alpha_{i j}$ is an arbitrary positive constant. The individual contributions $\frac{1}{2} Q_{i} u_{i}^{2}$ represents the $i$-th generator cost.

Assumption 3 Any optimal solution for the ED problem (28) has the property that the inequality constraint

$$
\left|\theta_{i}-\theta_{j}\right|<\alpha_{i j}<\frac{\pi}{2}, \quad \forall i \in \mathcal{B}, j \in \mathcal{N}_{i}
$$

is strictly satisfied.

Assumption 3 is intrinsic to the model of the network dynamics (2) with (3) [6], [2], which is used here. Under Assumption 3, the ED problem (28) can be simplified to a convex quadratic optimisation problem subject to a linear equality constraint, commonly recognised as Reduced Economic Dispatch (RED) [6] [31]:

$$
\operatorname{RED}\left\{\begin{aligned}
\min _{u} C(u) & :=\frac{1}{2} u^{T} Q u=\sum_{i \in \mathcal{G}} \frac{1}{2} Q_{i} u_{i}^{2} \\
\text { subject to } & \\
0 & =\underline{1}^{T} \hat{p}+\underline{1}^{T} u
\end{aligned}\right.
$$

Note that the linear constraint in (30) represents the lossless property of the power network, in which the total power generation matches the total power consumption [3]. The following Lemma holds

Lemma 1 The optimal solution $u^{\star}$ of the RED (30) is characterised by the identical marginal costs as follows [12]:

$$
Q_{i} u_{i}^{\star}=Q_{j} u_{j}^{\star}, \quad \forall i, j \in \mathcal{G}
$$

Furthermore, any strictly feasible solution of the ED (28) is optimal solution of the RED (30) [6]. The vector $\xi^{\star} \in \mathbb{R}^{|\mathcal{G}|}$ with all equal entries corresponding to the (optimal) identical marginal cost in (31), which is given by the relation [31]:

$$
\xi^{\star}=\frac{1 \underline{1}^{T} \hat{p}}{\underline{1}^{T} Q^{-1} \underline{1}}
$$

In the present paper it is assumed that the optimal value for $\xi^{\star}$ in (32) is computed by the same reference maker node in Assumption 2. It follows that the reference maker is governed by the algebraic equation (32). The optimal control input will be given by [6], [7]:

$$
u^{\star}=Q^{-1} \xi^{\star}
$$


The underlying idea is to use a SM control architecture which is capable of achieving the condition (33) in finite time, by using the information coming from the distributed observer scheme and the reference value coming from the reference maker node. It is proven that the frequency deviation asymptotically tends to zero in each generator node, thus achieving both frequency regulation and RED optimisation problem. Let the vector of deviation in marginal cost be $\Delta \xi:=\xi-\xi^{\star}$, where $\xi$ is the vector of marginal cost. A variant of dynamic control structure, originally proposed in [7], is utilised here to reduce the vector of deviation in marginal cost to zero in finite time, and as a consequence the optimal control input in (33) is attained in finite time. Compared with approaches in [7], [12], [22], it is the finite time convergence to the optimal control yielding optimal RED which is the originality of the proposed architecture.

Proposition 3 Suppose Assumption 3 and Lemma 1 hold, then, for (24), the dynamic control scheme

$$
\begin{aligned}
\Delta \dot{\xi} & =-W \Delta \xi+\Omega v-Q^{-1} \hat{\omega}_{\mathcal{G}} \\
u & =Q^{-1} \xi
\end{aligned}
$$

where $\xi \in \mathbb{R}^{|\mathcal{G}|}$ is the vector of marginal costs, $W=\operatorname{Diag}\left(W_{1}, \ldots, W_{|\mathcal{G}|}\right) \in \mathbb{R}^{|\mathcal{G}| \times|\mathcal{G}|}, \hat{\omega}_{\mathcal{G}}:=\operatorname{Col}\left(\hat{\omega}_{1}, \ldots, \hat{\omega}_{|\mathcal{G}|}\right)$, and $\Omega=\operatorname{Diag}\left(\Omega_{1}, \ldots, \Omega_{|\mathcal{G}|}\right) \in$ $\mathbb{R}^{|\mathcal{G}| \times|\mathcal{G}|}$ are positive definite design diagonal matrices, and the discontinuous injection term $v=\operatorname{Col}\left(\operatorname{sign}\left(\Delta \xi_{1}\right), \ldots, \operatorname{sign}\left(\Delta \xi_{|\mathcal{G}|}\right)\right)$, $v \in \mathbb{R}^{|\mathcal{G}|}$ has to be designed, ensures that:

I) $\Delta \xi \rightarrow 0$ and the RED (30) are satisfied in a finite time if each scalar component $\Omega_{i}$ of the matrix $\Omega$ satisfies

$$
\Omega_{i}>Q_{i}^{-1} \Lambda_{\omega}
$$

$\Lambda_{\omega}$ is a positive constant.

II) The frequency regulation is achieved in asymptotic sense.

Proof: I) Note that the dynamics in (34) are completely decentralised, and consequently each component $\Delta \xi_{i}$ does not depend on the other ones $\Delta \xi_{j}, j \neq i$. Therefore, it is possible to consider separately each scalar component

$$
\Delta \dot{\xi}_{i}=-W_{i} \Delta \xi_{i}-\Omega_{i} \operatorname{sign}\left(\Delta \xi_{i}\right)-Q_{i}^{-1} \hat{\omega}_{i}
$$

Note that in equation (36) the key-novelty is represented by the nonlinear term $\Omega_{i} \operatorname{sign}\left(\Delta \xi_{i}\right)$, which was not present in the earlier works [22], [23], [7], [31], where a similar structure for the dynamics in (36) was proposed. Consider a Lyapunov function

$$
V_{c_{i}}=\frac{1}{2} \Delta \xi_{i}^{2}
$$

Differentiating $V_{c_{i}}$ along the trajectory of the dynamics in (36) yields:

$$
\begin{aligned}
\dot{V}_{c_{i}} & =-W_{i}\left|\Delta \xi_{i}\right|^{2}-\Omega_{i}\left|\Delta \xi_{i}\right|-\Delta \xi_{i} Q_{i}^{-1} \hat{\omega}_{i} \\
& \leq-\Omega_{i}\left|\Delta \xi_{i}\right|+Q_{i}^{-1}\left|\hat{\omega}_{i}\right|\left|\Delta \xi_{i}\right|
\end{aligned}
$$

In order to guarantee negative definiteness of (38), one has to verify the following inequality

$$
\Omega_{i}>Q_{i}^{-1}\left|\hat{\omega}_{i}\right|
$$

or, in a more conservative way, it yields

$$
\Omega_{i}>Q_{i}^{-1} \Lambda_{\omega}
$$

It follows that $\dot{V}_{c_{i}} \leq-\eta_{i}\left|\Delta \xi_{i}\right|$ for some positive constant $\eta_{i}$. which implies $\Delta \xi_{i}=0$ in finite time. Therefore, each component $u_{i}=Q_{i}^{-1} \xi_{i}$ of the vector $u$ reaches in finite time the optimal value $u_{i}^{\star}$, which proves $\mathbf{I}$ ).

II) Note that the steady-state solution of (24) satisfying

$$
\begin{aligned}
\theta & =\theta^{\star} \\
\omega^{\star} & =\underline{0} \\
u^{\star} & =\nabla U_{\mathcal{G}}\left(\theta^{\star}\right)
\end{aligned}
$$

constitutes an optimality condition of equilibrium, as proven in [6]. The aim here is to show that the trajectories of the closed-loop system asymptotically converge to (41), by means of a Lyapunov function technique. Following [6] and [22], the closed-loop dynamics can be expanded around steady state:

$$
\begin{aligned}
\dot{\hat{\theta}}= & \hat{\omega} \\
M_{\mathcal{G}} \dot{\hat{\omega}}_{\mathcal{G}}= & -D_{\mathcal{G}} \hat{\omega}_{\mathcal{G}}+Q^{-1} \Delta \xi \\
& -\left(\nabla_{\mathcal{G}} U(\hat{\theta})-\nabla_{\mathcal{G}} U\left(\theta^{\star}\right)\right) \\
0= & -D_{\mathcal{L}} \hat{\omega}_{\mathcal{L}}-\left(\nabla_{\mathcal{L}} U(\hat{\theta})-\nabla_{\mathcal{L}} U\left(\theta^{\star}\right)\right) \\
\Delta \dot{\xi}= & -W \Delta \dot{\xi}+\Omega v-Q^{-1} \hat{\omega}_{\mathcal{G}}
\end{aligned}
$$


Consider the following energy storage function:

$$
V_{s}(x)=\frac{1}{2} \hat{\omega}_{\mathcal{G}}^{T} M_{\mathcal{G}} \hat{\omega}_{\mathcal{G}}+U(\hat{\theta})+\frac{1}{2} \Delta \xi^{T} \Delta \xi .
$$

where the auxiliary state vector $x:=\operatorname{Col}\left(\hat{\theta}, \hat{\omega}_{\mathcal{G}}, \Delta \xi\right)$. Under Assumption $3, V_{s}(x)$ is positive definite and strictly convex around the equilibrium point (41) [31] [6]. This can be easily verified by calculating the first and the second partial derivatives of $V_{s}(x)$. Analogously to [31], the energy function is shifted as follow

$$
V(x)=V_{s}(x)-V_{s}\left(x^{\star}\right)-\nabla V_{s}\left(x^{\star}\right)^{T}\left(x-x^{\star}\right)
$$

where the auxiliary state vector $x^{\star}:=\operatorname{Col}\left(\theta^{\star}, 0,0\right)$ denotes the equilibrium point in (41). Since $\nabla V_{s}\left(x^{\star}\right)=\operatorname{Col}\left(\nabla U\left(\theta^{\star}\right), 0,0\right)$, equation (44) can be rewritten as

$$
\begin{aligned}
V(x)= & \frac{1}{2} \hat{\omega}_{\mathcal{G}}^{T} M_{\mathcal{G}} \hat{\omega}_{\mathcal{G}}+U(\hat{\theta})-U\left(\theta^{\star}\right) \\
& -\nabla U\left(\theta^{\star}\right)^{T}\left(\hat{\theta}-\theta^{\star}\right)+\frac{1}{2} \Delta \xi^{T} \Delta \xi .
\end{aligned}
$$

As proven in [31] and [7], $V(x)$ is convex and positive definite with respect to the equilibrium point (41). The time derivative of $V(x)$ along the trajectories of the closed-loop system in (42) yields:

$$
\begin{aligned}
\dot{V}(x)= & -\hat{\omega}_{\mathcal{G}}^{T} D_{\mathcal{G}} \hat{\omega}_{\mathcal{G}}+\hat{\omega}_{\mathcal{G}}^{T} Q^{-1} \Delta \xi-\hat{\omega}_{\mathcal{G}}^{T} \nabla_{\mathcal{G}} U(\hat{\theta}) \\
& +\hat{\omega}_{\mathcal{G}}^{T} \nabla_{\mathcal{G}} U\left(\theta^{\star}\right)+\hat{\omega}^{T} \nabla U(\hat{\theta})-\nabla U\left(\theta^{\star}\right)^{T} \hat{\omega} \\
& -\Delta \xi^{T} W \Delta \xi+\Delta \xi^{T} \Omega v-\Delta \xi^{T} Q^{-1} \hat{\omega}_{\mathcal{G}}
\end{aligned}
$$

From the developments in part I) of Proposition 3, the inequality $-\Delta \xi^{T} W \Delta \xi+\Delta \xi^{T} \Omega v<0$ After straightforward algebraic manipulations, the inequality

$$
\begin{aligned}
\dot{V}(x) \leq & -\hat{\omega}_{\mathcal{G}}^{T} D_{\mathcal{G}} \hat{\omega}_{\mathcal{G}}-\hat{\omega}_{\mathcal{G}}^{T} \nabla_{\mathcal{G}} U(\hat{\theta})+\hat{\omega}^{T} \nabla U(\hat{\theta}) \\
& +\hat{\omega}_{\mathcal{G}}^{T} \nabla_{\mathcal{G}} U\left(\theta^{\star}\right)-\nabla U\left(\theta^{\star}\right)^{T} \hat{\omega}
\end{aligned}
$$

is obtained. By exploiting the structure of the right-hand side of (47), and the substitutions

$$
\begin{aligned}
\hat{\omega}_{\mathcal{L}}^{T} \nabla_{\mathcal{L}} U(\hat{\theta}) & =-\hat{\omega}_{\mathcal{G}}^{T} \nabla_{\mathcal{G}} U(\hat{\theta})+\hat{\omega}^{T} \nabla U(\hat{\theta}) \\
-\hat{\omega}_{\mathcal{L}}^{T} \nabla_{\mathcal{L}} U\left(\theta^{\star}\right) & =\hat{\omega}_{\mathcal{G}}^{T} \nabla_{\mathcal{G}} U\left(\theta^{\star}\right)-\nabla U\left(\theta^{\star}\right)^{T} \hat{\omega}
\end{aligned}
$$

it can be verified that

$$
\dot{V}(x) \leq-\hat{\omega}_{\mathcal{G}}^{T} D_{\mathcal{G}} \hat{\omega}_{\mathcal{G}}+\hat{\omega}_{\mathcal{L}}^{T}\left(\nabla_{\mathcal{L}} U(\hat{\theta})-\nabla_{\mathcal{L}} U\left(\theta^{\star}\right)\right)
$$

Moreover, given (42), the vector $\hat{\omega}_{\mathcal{L}}^{T}$ can be shown to be equal to:

$$
\hat{\omega}_{\mathcal{L}}^{T}=\left(\nabla_{\mathcal{L}} U\left(\theta^{\star}\right)-\nabla_{\mathcal{L}} U(\hat{\theta})\right) D_{\mathcal{L}}^{-1}
$$

Note that in (50) the invertibility property of $D_{\mathcal{L}}$, which is ensured by construction, is used. Substituting for $\hat{\omega}_{\mathcal{L}}^{T}$ in $(49)$

$$
\begin{aligned}
\dot{V}(x) \leq & -\hat{\omega}_{\mathcal{G}}^{T} D_{\mathcal{G}} \hat{\omega}_{\mathcal{G}} \\
& -\left(\nabla_{\mathcal{L}} U(\hat{\theta})-\nabla_{\mathcal{L}} U\left(\theta^{\star}\right)\right)^{T} D_{\mathcal{L}}^{-1}\left(\nabla_{\mathcal{L}} U(\hat{\theta})-\nabla_{\mathcal{L}} U\left(\theta^{\star}\right)\right)
\end{aligned}
$$

It follows that $\dot{V}<0$. Therefore, the Lyapunov function is strictly decreasing excluding the equilibrium point (41). It follows that the closed-loop system trajectories asymptotically converges to (41), which prove the proposition.

Remark 5 Note that the SM scheme (36) brings benefits in terms of robustness to possible disturbances and attacks. To better illustrate this, consider equation (36) rewritten as follows

$$
\Delta \dot{\xi}_{i}=-W_{i} \Delta \xi_{i}-\Omega_{i} \operatorname{sign}\left(\Delta \xi_{i}\right)-Q_{i}^{-1} \hat{\omega}_{i}+z_{i}
$$

where $z_{i}$ is a possible disturbance or external attack. It is assumed that $\left|z_{i}\right|<\Delta_{z_{i}}$, where $\Delta_{z_{i}}$ is a known positive constant. As $z_{i}$ is a bounded matched disturbance, $\Delta \xi_{i}$ can still be driven to zero in finite time, thus addressing the RED in (30).

\section{Simulation Case Study}

In this section the performance of the proposed scheme for frequency regulation and ED are evaluated by using the IEEE 39 bus benchmark [32], [33]. This power network, which has been used in other relevant works (see, e.g., [6], [23], and [22]), is composed of an interconnection of 10 generator buses and 29 load buses, as shown in the schematic in Figure 1. The numerical data for this benchmark is reported in [33]. As in [6], [23], and [22], the droop control coefficients are set equal to $D_{i}=1$ (p.u.) $\forall i=1, \ldots, 39$, and during the simulation, for time $t \geq 1$ seconds, the power demand increases by 0.33 (p.u.) at the 4-th, 12-th and 20-th bus. The base power of the network is set equal to 1 (p.u.)= 100 (MW). 


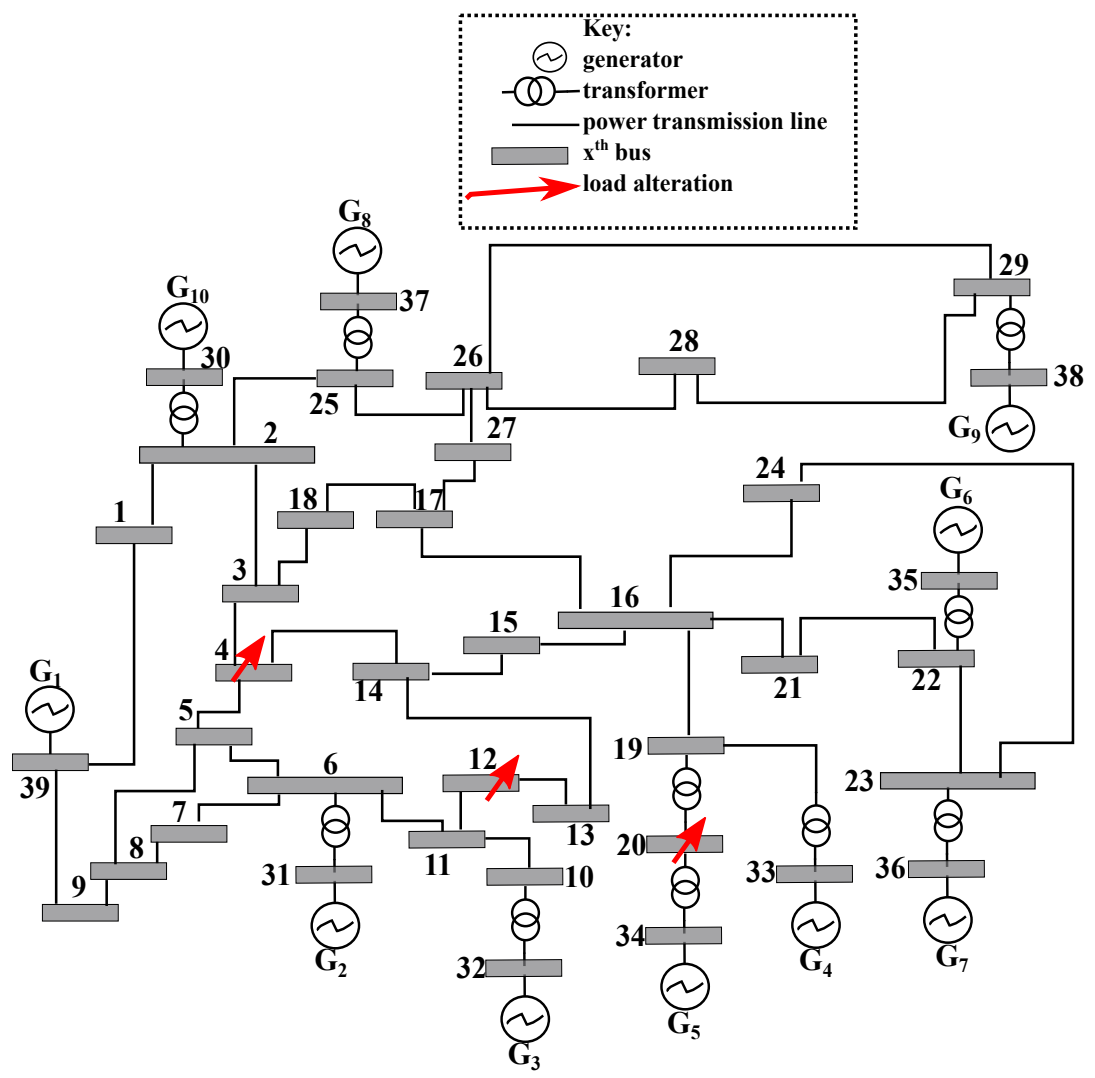

Figure 1. A schematic of the IEEE 39 bus benchmark.
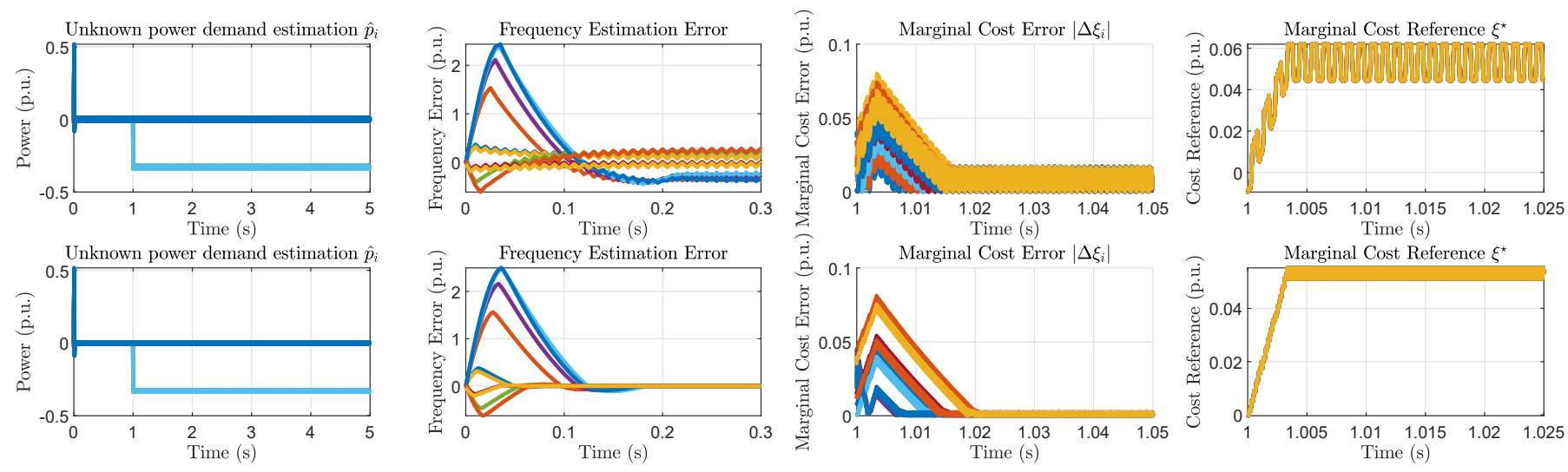

Figure 2. From the left: time evolution of the estimates $\hat{p}_{i}$ of the unknown power demands; time evolution of the frequency estimation error $e_{\omega_{i}}$; time evolution of the marginal cost error during the time interval $[1,1.05]$; time evolution of the optimal marginal cost reference during the time interval $[1,1.05]$ seconds, in accordance with (32). (a): Scenario with noise; (b): Noise-free scenario 

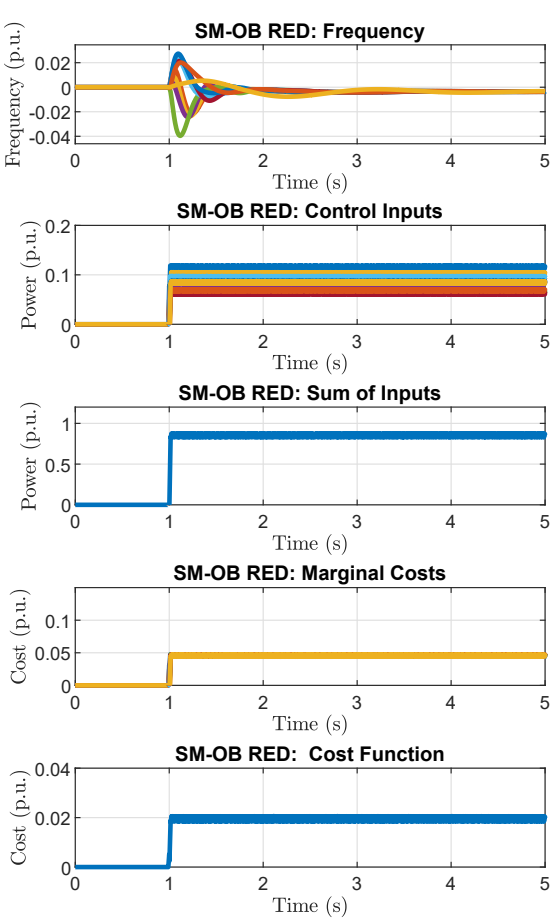

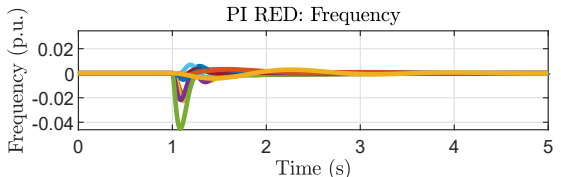

PI RED: Control Inputs

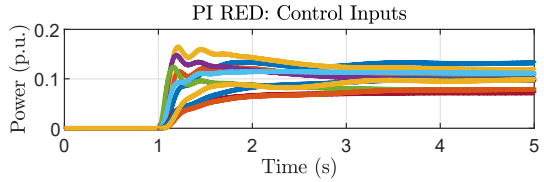

PI RED: Sum of Inputs

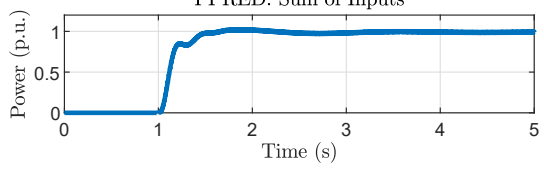

PI RED: Marginal Costs

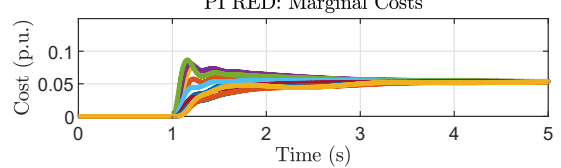

PI RED: Cost Function

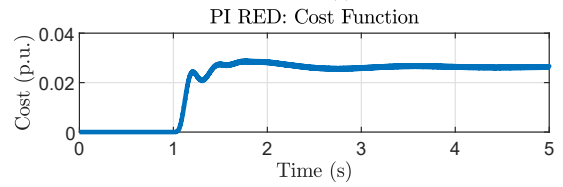

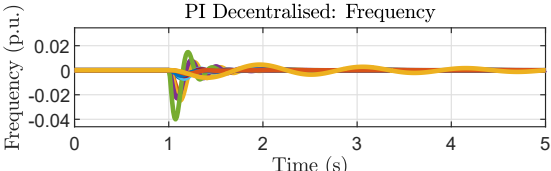

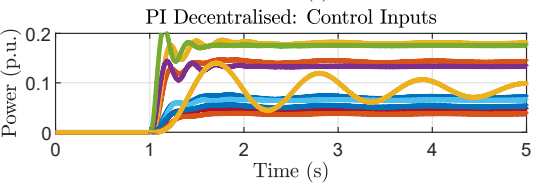

PI Decentralised: Sum of Inputs

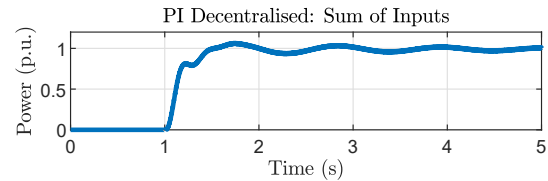

PI Decentralised: Marginal Costs

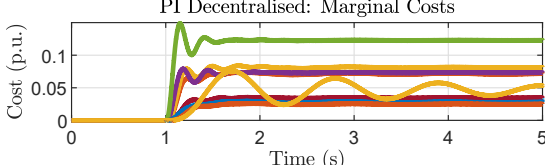

PI Decentralised: Cost Function

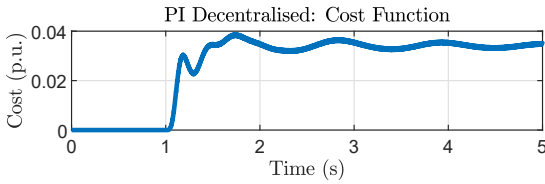

Figure 3. A comparison of the proposed observer-based control scheme, with the pre-existing PI RED and PI Decentralised approaches. From the top, the following key-indicators are considered: frequency deviations, control inputs, sum of control inputs, marginal costs, cost function.

The generator observer design parameters are $k_{g_{i}}=21, k_{g_{i}}=214, k_{g_{i}}=250, \forall i=1, \ldots 10$, whilst the load observer gains are $k_{l_{l_{i}}}=15, k_{2_{l_{i}}}=110, \forall i=1, \ldots, 29$. ED design parameters are: $Q=\operatorname{Diag}([0.4,0.0,0.45,0.55,0.7,0.48,0.74,0.53,0.67,0.54])$, and $W=20 I_{(10 \times 10)}$. The power network benchmark, together with the proposed observer-based control scheme is implemented in a Matlab-Simulink R2018a environment. The voltage phase angle measurements were acquired at a frequency of $120(\mathrm{~Hz})$, which is compatible with a PMU's features [2]. The fixed integration step size Odel (Euler method) was employed for the SM observers implementation, with an integration step size equal to 50 microseconds. To achieve this, a Zero Order Hold $(\mathrm{ZOH})$ architecture for the PMU output was used, together with a cubic spline to accommodate the faster update rate for the observer calculations. A comparison based on this case study is undertaken: SM-OBS RED denotes the proposed SM observer-based control scheme for frequency regulation and the ED problem; PI-RED denotes the distributed Proportional Integral (PI) control scheme for the frequency regulation and RED problem as formulated in [6]; PI-Decentralised denotes a conventional decentralised PI control scheme for frequency regulation. A band-limited measurement noise $n$ is introduced in each bus of the network in equation (24) with $|n| \leq 0.1$ (mrad). In this situation $y=\theta+n$ in (24). Figure 2 shows that the proposed scheme has the ability to estimate in finite time the unknown power demand variation $\hat{p}_{i}$, which is equal to 0.33 (p.u.) at the 4-th, 12-th and 20-th bus. The negative sign means that the power is associated with an increase of consumption at those buses. The frequency estimation errors are steered to zero in finite time. In addition, the marginal cost errors are driven to zero in finite time, thus tracking the marginal cost reference. The impact of the measurement noise is shown in Figure 2(a). Specifically, the power demand and the frequencies can still be estimated with acceptable accuracy, whilst the marginal cost reference is affected by small fluctuations. Figure 3 shows an extensive comparison between the proposed scheme and the two well-established control architecture described earlier. The frequency is asymptotically steered to zero by all the three control architectures. The control inputs counterbalance the growth of power demand. The proposed scheme is characterised by faster controller dynamics, which are able to reach the optimal value in finite time. This results in a perfect counterbalance of 1 (p.u.). The marginal costs reach the optimal value in finite time (few seconds) by using the proposed SM-OBS RED, whilst the optimality is asymptotically achieved via PI-RED scheme. In contrast, the decentralised PI architecture is not able to reach the ED requirement.

\section{CONCLUSION}

In this paper a novel SM observer-based scheme has been formulated to achieve frequency regulation and ED in structurepreserving power grids composed of an interconnection of generators and load buses. A network of SM observers has been proposed to estimate the unmeasured frequency deviation in generator buses, and to track the unknown power demand in all the buses in finite time. A SM control architecture was proposed to achieve in finite time the optimal marginal cost for the 
generator buses. The numerical simulation test case, implemented on the widely used IEEE 39 bus benchmark, has revealed that the proposed scheme displays better performances when compared with existing methods.

\section{REFERENCES}

[1] A. S. Debs, Modern power systems control and operation. Springer Science \& Business Media, 2012.

[2] P. W. Sauer, M. A. Pai, and J. H. Chow, Power system dynamics and stability: with synchrophasor measurement and power system toolbox. John Wiley \& Sons, 2017

[3] P. Kundur, N. J. Balu, and M. G. Lauby, Power system stability and control. McGraw-hill New York, 1994, vol. 7.

[4] D. K. Molzahn, F. Dörfler, H. Sandberg, S. H. Low, S. Chakrabarti, R. Baldick, and J. Lavaei, "A survey of distributed optimization and control algorithms for electric power systems," IEEE Transactions on Smart Grid, vol. 8, no. 6, pp. 2941-2962, 2017.

[5] B. H. Chowdhury and S. Rahman, "A review of recent advances in economic dispatch," IEEE Transactions on Power Systems, vol. 5, no. 4, pp. $1248-1259,1990$.

[6] C. Zhao, E. Mallada, and F. Dörfler, "Distributed frequency control for stability and economic dispatch in power networks," in Proc. of American Control Conference (ACC), Chicago, IL, USA, July 2015, pp. 2359-2364.

[7] S. Trip, M. Bürger, and C. De Persis, "An internal model approach to (optimal) frequency regulation in power grids with time-varying voltages," Automatica, vol. 64, pp. 240-253, 2016.

[8] C. Zhao, U. Topcu, N. Li, and S. Low, "Design and stability of load-side primary frequency control in power systems," IEEE Transactions on Automatic Control, vol. 59, no. 5, pp. 1177-1189, 2014.

[9] C. Zhao, E. Mallada, S. H. Low, and J. Bialek, "Distributed plug-and-play optimal generator and load control for power system frequency regulation," International Journal of Electrical Power and Energy Systems, vol. 101, pp. 1-12, 2018.

[10] F. Guo, C. Wen, J. Mao, J. Chen, and Y.-D. Song, "Hierarchical decentralized optimization architecture for economic dispatch: A new approach for large-scale power system," IEEE Transactions on Industrial Informatics, vol. 14, no. 2, pp. 523-534, 2018.

[11] C. Edwards and S. Spurgeon, Sliding mode control: theory and applications. CRC Press, 1998.

[12] S. Trip, M. Cucuzzella, C. De Persis, A. van der Schaft, and A. Ferrara, "Passivity-based design of sliding modes for optimal load frequency control," IEEE Transactions on Control Systems Technology, no. 99, pp. 1-14, 2018.

[13] K. Vrdoljak, N. Perić, and I. Petrović, "Sliding mode based load-frequency control in power systems," Electric Power Systems Research, vol. 80, no. 5 , pp. 514-527, 2010.

[14] C. P. Tan and C. Edwards, "Sliding mode observers for robust detection and reconstruction of actuator and sensor faults," International Journal of Robust and Nonlinear Control: IFAC-Affiliated Journal, vol. 13, no. 5, pp. 443-463, 2003.

[15] G. Rinaldi, P. P. Menon, C. Edwards, and A. Ferrara, "Sliding mode based dynamic state estimation for synchronous generators in power systems," IEEE Control Systems Letters, vol. 2, no. 4, pp. 785-790, 2018.

[16] G. Rinaldi, M. Cucuzzella, and A. Ferrara, "Third order sliding mode observer-based approach for distributed optimal load frequency control," IEEE Control Systems Letters, vol. 1, no. 2, pp. 215-220, 2017.

[17] G. Rinaldi, P. P. Menon, C. Edwards, and A. Ferrara, "Design and validation of a distributed observer-based estimation scheme for power grids," IEEE Transactions on Control Systems Technology, 2018.

[18] C. Mellucci, P. P. Menon, C. Edwards, and A. Ferrara, "Second-order sliding mode observers for fault reconstruction in power networks," IET Control Theory \& Applications, vol. 11, no. 16, pp. 2772-2782, 2017.

[19] E. Scholtz, R. F. Nuqui, D. Julian, and A. R. Grightmire, "Real-time power-line sag monitoring using time-synchronized power system measurements," Nov. 17 2009, uS Patent 7,620,517.

[20] A. R. Bergen and D. J. Hill, "A structure preserving model for power system stability analysis," IEEE Transactions on Power Apparatus and Systems, no. 1 , pp. $25-35,1981$.

[21] K. Liao and Y. Xu, "A robust load frequency control scheme for power systems based on second-order sliding mode and extended disturbance observer," IEEE Transactions on Industrial Informatics, vol. 14, no. 7, pp. 3076-3086, 2018.

[22] F. Dörfler and S. Grammatico, "Gather-and-broadcast frequency control in power systems," Automatica, vol. 79, pp. $296-305,2017$.

[23] K. Xi, J. L. Dubbeldam, H. X. Lin, and J. H. van Schuppen, "Power-imbalance allocation control of power systems-secondary frequency control," Automatica, vol. 92, pp. 72-85, 2018.

[24] V. Vittal, J. D. McCalley, P. M. Anderson, and A. Fouad, Power system control and stability. John Wiley \& Sons, 2019.

[25] A. Chalanga, S. Kamal, L. M. Fridman, B. Bandyopadhyay, and J. A. Moreno, "Implementation of super-twisting control: Super-twisting and higher order sliding-mode observer-based approaches," IEEE Transactions on Industrial Electronics, vol. 63, no. 6, pp. 3677-3685, 2016.

[26] F. A. Ortiz-Ricardez, T. Sánchez, and J. A. Moreno, "Smooth Lyapunov function and gain design for a second order differentiator," in Proc. of 54th IEEE Conference on Decision and Control (CDC), Osaka, Japan, December 2015, pp. 5402-5407.

[27] E. Cruz-Zavala and J. A. Moreno, "Levant's arbitrary order exact differentiator: A Lyapunov approach," IEEE Transactions on Automatic Control, 2018.

[28] G. Rinaldi, P. P. Menon, C. Edwards, and A. Ferrara, "Distributed super-twisting sliding mode observers for fault reconstruction and mitigation in power networks," in Proc. of 57th IEEE Conference on Decision and Control (CDC), Miami Beach, FL, USA, December 2018, pp. 5550-5555.

[29] J. A. Moreno and M. Osorio, "Strict Lyapunov functions for the super-twisting algorithm," IEEE Transactions on Automatic Control, vol. 57, no. 4, pp. $1035-1040,2012$.

[30] R. J. Davy and I. A. Hiskens, "Lyapunov functions for multimachine power systems with dynamic loads," IEEE Transactions on Circuits and Systems I: Fundamental Theory and Applications, vol. 44, no. 9, pp. 796-812, 1997.

[31] P. Monshizadeh, C. De Persis, T. Stegink, N. Monshizadeh, and A. van der Schaft, "Stability and frequency regulation of inverters with capacitive inertia," in Proc. of IEEE 56- th Annual Conference on Decision and Control (CDC). Melbourne, Australia, December 2017, pp. 5696-5701.

[32] A. Moeini, I. Kamwa, P. Brunelle, and G. Sybille, "Open data ieee test systems implemented in simpowersystems for education and research in power grid dynamics and control," in Proc. of 50- th International Universities Power Engineering Conference (UPEC), Staffordshire University, UK, September 2015, pp. 1-6.

[33] I. Hiskens, "IEEE PES task force on benchmark systems for stability controls," Technical Report, 2013. 